\title{
Thymic epithelial tumors and metastasis to the brain: a case series and systematic review
}

\author{
Helen Gharwan ${ }^{1 *}$, Chul Kim ${ }^{1 *}$, Anish Thomas ${ }^{1}$, Arlene Berman ${ }^{1}$, Sun A. Kim ${ }^{2}$, Nadia Biassou ${ }^{3}$, Seth $M$. \\ Steinberg ${ }^{4}$, Arun Rajan ${ }^{1}$ \\ ${ }^{1}$ Thoracic and Gastrointestinal Oncology Branch, ${ }^{2}$ Laboratory of Pathology, ${ }^{3}$ Division of Neuroradiology, Department of Imaging Sciences, \\ ${ }^{4}$ Biostatistics and Data Management Section, Center for Cancer Research, National Cancer Institute, National Institutes of Health, Bethesda, \\ Maryland, USA \\ Contributions: (I) Conception and design: H Gharwan, C Kim, A Rajan; (II) Administrative support: C Kim, A Rajan; (III) Provision of study \\ materials or patients: H Gharwan, C Kim, A Thomas, A Berman, A Rajan; (IV) Collection and assembly of data: All authors; (V) Data analysis and \\ interpretation: All authors; (VI) Manuscript writing: All authors; (VII) Final approval of manuscript: All authors. \\ *These authors contributed equally to the work. \\ Correspondence to: Arun Rajan, MD. Thoracic and Gastrointestinal Oncology Branch, National Cancer Institute, National Institutes of Health, 10 \\ Center Drive, Bldg. 10, Room 4-5330, Bethesda, Maryland 20892-1906, USA. Email: rajana@mail.nih.gov.
}

Background: Thymic epithelial tumors (TETs) rarely metastasize to the brain. Clinico-pathologic features of TET patients with brain metastasis are not well described.

Methods: TET patients referred for consultation or screening for clinical trials are included. Imaging to evaluate for brain metastases was performed when clinically indicated or if required for screening. Tumor tissue from brain metastases was obtained for analysis, when available. Clinical characteristics and survival was evaluated and a systematic review of the literature on brain metastases associated with TETs was performed.

Results: Fourteen TET patients with brain metastasis were identified. Median age at TET diagnosis was 53 years (range: $31-71$ years). Twelve patients had thymic carcinoma and two patients had World Health Organization B3 thymoma. Median time from TET diagnosis to discovery of brain metastases was 2.5 years (range: 9 months-8.3 years). Eleven patients had extracranial, extrathoracic metastases during presentation with brain metastases. Three patients underwent surgery and radiation therapy, eight patients received radiation therapy alone, and one patient had surgery alone. One patient with thymoma died 11 months after diagnosis of brain metastases and another patient died but with unknown date of diagnosis of brain metastases. Among 12 patients with thymic carcinoma, 11 of whom had a known date of brain metastases diagnosis, the median potential follow-up is 35.8 months, and median overall survival (OS) from diagnosis of brain metastases is 13.1 months.

Conclusions: Although uncommon, patients with advanced thymic carcinoma can develop brain metastases. Appropriate imaging and aggressive treatment should be considered for these patients.

Keywords: Thymoma; thymic carcinoma; brain; central nervous system; metastasis

Submitted Jul 05, 2017. Accepted for publication Aug 17, 2017.

doi: $10.21037 /$ tlcr.2017.08.06

View this article at: http://dx.doi.org/10.21037/tlcr.2017.08.06

\section{Introduction}

Thymic epithelial tumors (TETs) are very rare (1) with an incidence of 0.15 per 100,000 person-years. Thymic carcinomas account for less than $5 \%$ of these cases (2). Metastases to extrathoracic sites are uncommon (1.5-15.5\%) $(3,4)$. Metastasis to the brain is very rare compared with other solid tumors, where brain metastasis is reported to 
occur in $25-50 \%$ of all patients [e.g., in $50-60 \%$ of patients with lung cancer, $15-20 \%$ of patients with breast cancer or $5-10 \%$ of patients with malignant melanoma (5)]. Brain metastasis from common solid tumors portends a poor prognosis with median overall survival (OS) of 3-4 months $(6,7)$, but due to limited data on the incidence and prevalence of brain metastases in TETs, the natural history of disease and prognosis is unclear. Brain involvement has, however, been reported to be associated with worse prognosis and short survival of a few months (8).

In this single-institution case series, we describe the clinical characteristics of patients with TETs and brain metastasis, provide a systematic review of the literature on this topic, and offer recommendations for the management of these rare cases.

\section{Methods}

Patients with histologically confirmed TETs and documented presence of brain metastasis, either based on historical records or diagnosed during evaluation for enrollment in clinical trials, are included in this report. All patients provided written informed consent for participation in a clinical protocol (NCT00026754) approved by the institutional review board at the National Cancer Institute. The following clinical characteristics were recorded for further analysis: age, gender, TET subtype, metastatic sites, clinical symptoms, location of brain metastasis, interval between primary TET diagnosis and brain metastasis, treatment received and length of survival. Where available, tissue from surgically resected brain tumors was obtained and confirmed by a pathologist (SAK) to be a metastasis from the original primary TET. In patients with thymic carcinoma who have a known date of diagnosis of brain metastases, univariate survival analysis was performed using the Kaplan-Meier method. Survival was determined from the date of development of brain metastases until date of death or last follow-up. The statistical significance of the difference in survival curves was determined by an exact log-rank test. Patients were divided into groups based on gender, age at brain metastasis diagnosis, interval from TET diagnosis until diagnosis of brain metastasis, number of brain metastases, and location of other metastases. All P values are two-tailed and reported without adjustment for multiple comparisons. In view of the very small number of patients included in this study, the $\mathrm{P}$ values should be interpreted with caution as being very tentative.
A systematic search was performed on TETs and brain metastasis. MEDLINE (January 1985 to October 2016) English language abstracts were searched using the search term including the following key words: thymoma, thymic tumor, TET, thymic cancer, thymic carcinoma, brain metastasis, and intracranial metastasis. A manual search of the references of relevant studies was also performed. Reviews of the titles and abstracts of 301 articles, followed by review of the full text of potentially relevant articles, identified 22 cases of TETs metastatic to the brain.

\section{Results}

Between January 2009 and December 2014, 14 patients with TET were found to have brain metastasis; 8 were males and 6 females. Clinico-pathologic features are summarized in Table 1. The median age at TET diagnosis was 53 years. For the 12 patients with a known date of brain metastases, the median time from initial diagnosis to discovery of brain involvement was 2.5 years (range: 9 months -8.3 years). Twelve patients had thymic carcinoma with the most common histologic subtype being squamous cell carcinoma $(n=7)$. Two patients had primary tumors classified as WHO subtype B3 thymoma. The mean number of systemic therapies prior to diagnosis of brain metastasis was 3.3 (range: 1-9). At the time of diagnosis of brain metastasis, 11 of $14(78.6 \%)$ patients had extrathoracic disease, involving bones $(\mathrm{n}=8 / 11)$, liver $(\mathrm{n}=7 / 11)$, lymph nodes $(n=6 / 11)$ and kidney $(n=1 / 11)$. Three patients had exclusively intrathoracic involvement.

Patients presented with diverse neurologic symptoms such as headache, vision change, mental status change, memory loss, speech difficulty, and seizures before the diagnosis of brain metastasis was made (Table 2) or were asymptomatic at presentation with incidental discovery of brain metastases. Among eight patients whose brain imaging was available for review, most patients $(n=6 / 8 ; 75 \%)$ had a single brain metastasis at the time of diagnosis of brain involvement with size ranging from $6 \mathrm{~mm}$ to 5.2 $\mathrm{cm}$. Figure 1 shows various radiographic presentation of brain metastasis. The frontal lobe was the most frequently involved location $(n=6 / 8)$, followed by the parietal lobe $(n=3 / 8)$, cerebellum $(n=3 / 8)$, temporal and occipital lobes $(\mathrm{n}=2 / 8)$. One patient who presented with blurry vision was found to have metastasis to the retina (Figure 1E).

Eight patients were treated with radiotherapy alone, 
Table 1 Clinico-pathologic characteristics of patients with TETs and brain metastases

\begin{tabular}{|c|c|}
\hline Characteristics & Data $(\mathrm{N}=14)$ \\
\hline \multicolumn{2}{|l|}{ Age at TET diagnosis } \\
\hline Median & 53 \\
\hline Range & $31-71$ \\
\hline \multicolumn{2}{|l|}{ Age at brain metastasis diagnosis } \\
\hline Median & 57.5 \\
\hline Range & $34-72$ \\
\hline \multicolumn{2}{|l|}{ Sex, No. (\%) } \\
\hline Male & $8(57.1)$ \\
\hline Female & $6(42.9)$ \\
\hline \multicolumn{2}{|l|}{ Pathology, No. (\%) } \\
\hline Thymic carcinoma & $12(85.7)$ \\
\hline Thymoma, WHO subtype B3 & $2(14.3)$ \\
\hline \multicolumn{2}{|c|}{ Histologic subtypes of thymic carcinoma, No. (\%) } \\
\hline Squamous cell carcinoma & $7(58.3)$ \\
\hline Neuroendocrine & $1(8.3)$ \\
\hline Basaloid carcinoma & $1(8.3)$ \\
\hline Unclassified & $3(25.0)$ \\
\hline \multicolumn{2}{|c|}{$\begin{array}{l}\text { Location of extrathoracic metastatic lesions at time of brain } \\
\text { metastasis, No. (\%) }\end{array}$} \\
\hline Bone & $8(57.1)$ \\
\hline Lymph nodes & $7(50.0)$ \\
\hline Liver & $6(42.9)$ \\
\hline Kidney & $1(7.1)$ \\
\hline
\end{tabular}

Treatment prior to diagnosis of brain metastasis, No. (\%)

$\begin{array}{lc}\text { Surgery } & 9(64.3) \\ \text { Radiation } & 8(61.5) \\ \text { Systemic treatment } & 14(100.0)\end{array}$

Number of systemic treatments prior to brain metastasis diagnosis

$\begin{array}{ll}\text { Mean } & 3.3 \\ \text { Range } & 1-9\end{array}$

TET, thymic epithelial tumor. including stereotactic radiosurgery (SRS) in three patients, and whole brain radiotherapy (WBRT) in two patients. The type of radiotherapy is unclear in three cases. Two patients were treated with surgery and radiotherapy and one patient underwent surgery alone. The treatment received for brain metastasis is unclear in two patients. After treatment of brain metastasis, some patients received additional therapy for systemic disease, including cisplatin/etoposide, carboplatin/paclitaxel, temozolomide, capecitabine/ gemcitabine, and sunitinib.

Histological analysis of the brain metastasis was possible in three cases, and confirmed findings consistent with the patient's primary tumor (Figures 2,3).

A total of 8 of the 14 patients have died. One patient with thymoma died approximately 11 months after diagnosis of brain metastasis. The other patient with thymoma died at an unknown interval after the diagnosis of brain metastases. Among the 12 patients with thymic carcinoma, 11 of whom have known dates of diagnosis of brain metastases, the median potential follow-up is 35.8 months from the date of diagnosis of brain metastases, and the median survival is 13.1 months (95\% CI: 1.7 months to undefined). Univariate analyses in the patients with thymic carcinoma to explore possible associations with prognostic factors revealed that solitary ( $v s$. multiple) brain metastasis was potentially associated with improved OS based on only two patients with multiple brain metastases, but survival was not associated with gender, age at brain metastasis diagnosis, interval from TET diagnosis until diagnosis of brain metastases, and location of other metastases (all P>0.20). Median OS was not reached in patients with solitary brain metastasis, with one death at 9.9 months among 6 patients (12 months OS $=80 \%$, 95\% CI: 20.4-96.9\%). Of two patients with multiple brain metastases, one death occurred at 9 months and one patient is alive after 1 month follow-up.

A total of 22 cases of brain metastases from TETs were found in the literature (Table 3). The median age at diagnosis was 48.5 years. Six $(27.3 \%)$ cases occurred in females and the rest $(72.7 \%)$ in males. About two thirds $(n=14 / 22)$ of patients had brain metastases from thymic carcinoma. Eight cases of brain metastases from thymoma are reported involving all histological subtypes from type A to B3. Thirteen (59.1\%) patients with brain metastases 
Table 2 Treatment histories of patients with TETs and brain metastases

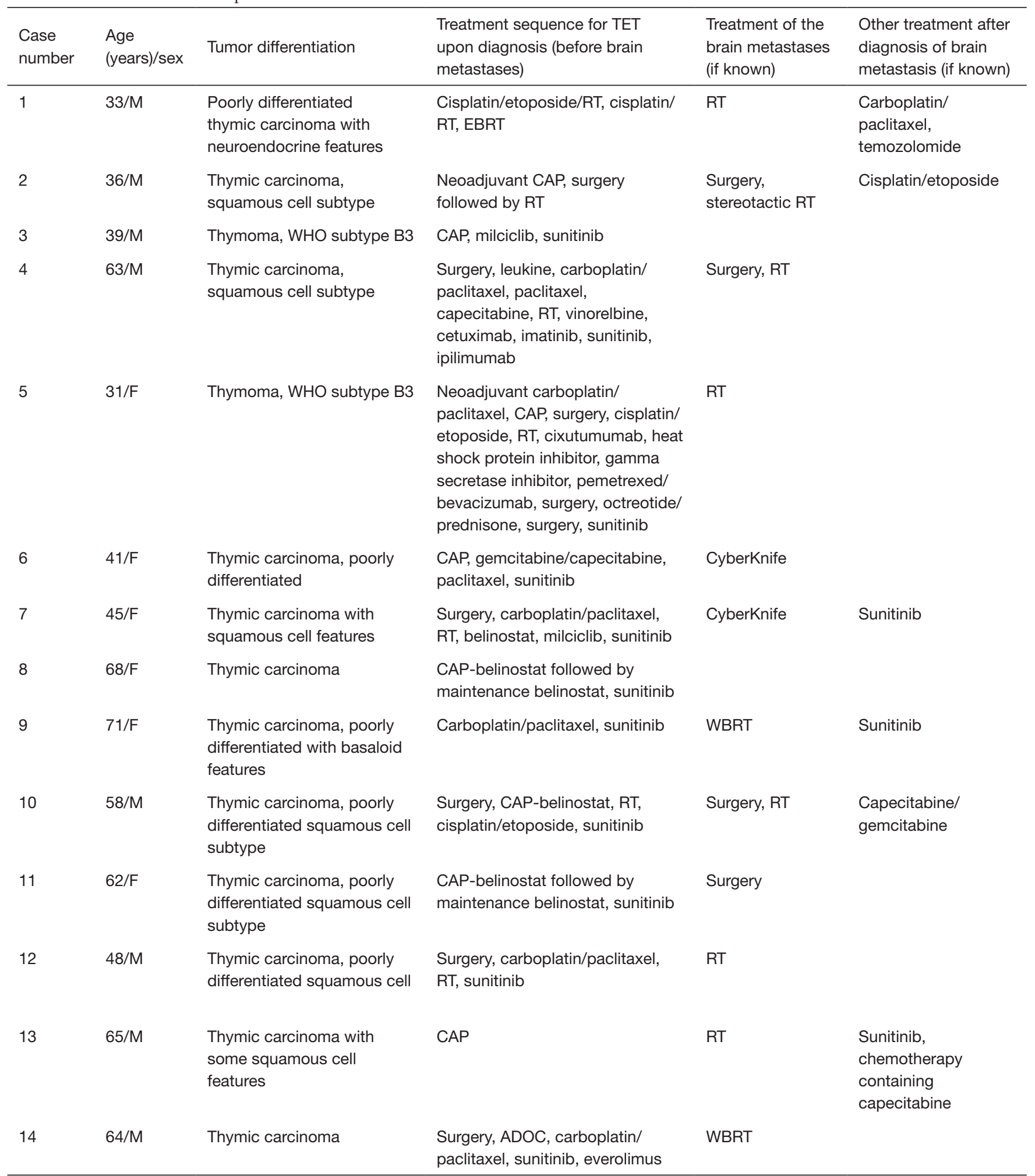

ADOC, cisplatin, doxorubicin, vincristine, cyclophosphamide; CAP, cisplatin, doxorubicin, cyclophosphamide; EBRT, external beam radiation therapy; F, female; $\mathrm{M}$, male; $\mathrm{RT}$, radiation therapy; $\mathrm{SRT}$, stereotactic radiotherapy; WBRT, whole brain radiotherapy; WHO, World Health Organization. 

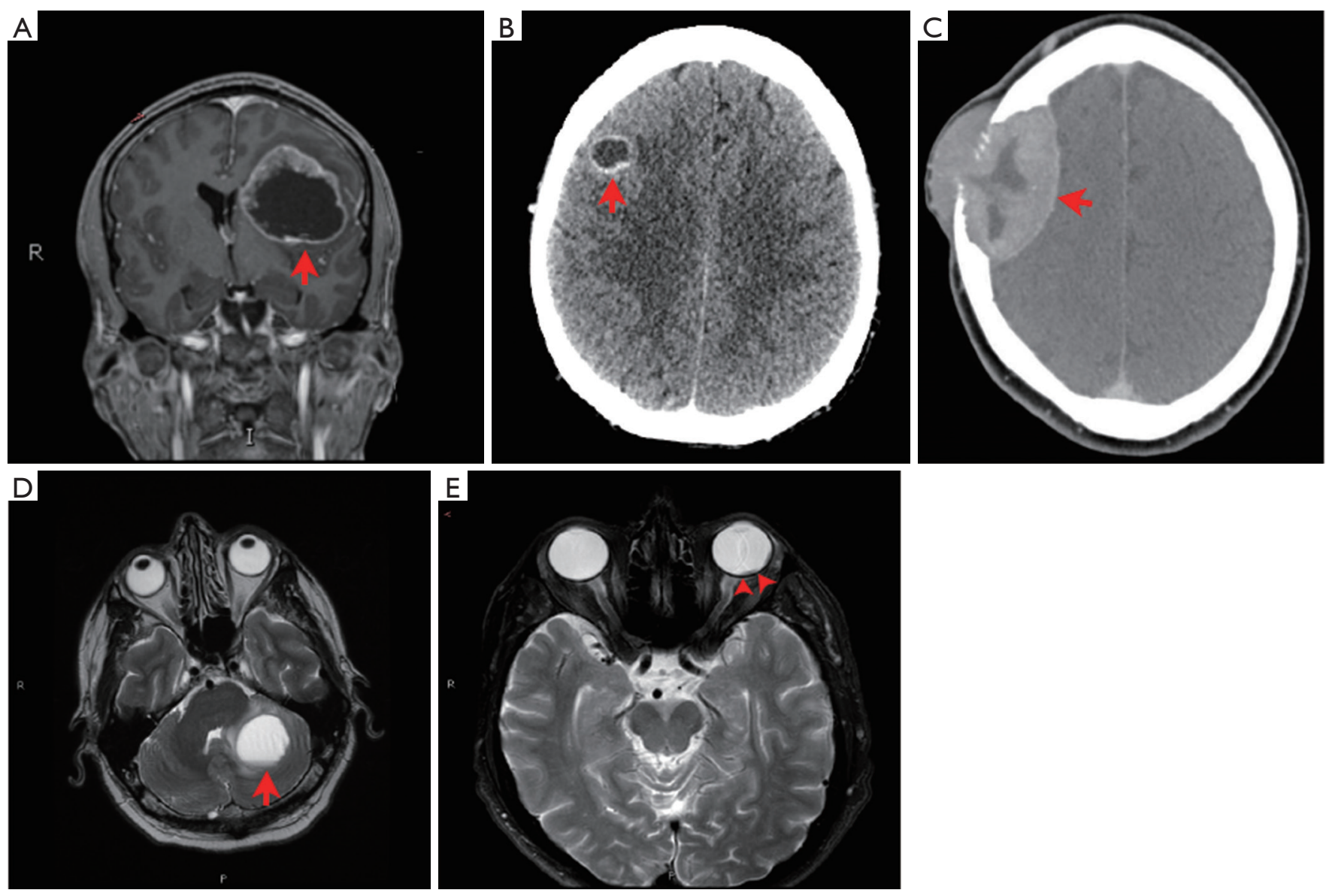

Figure 1 Spectrum of radiographic presentation of brain metastasis from thymic carcinoma. (A) Case 1: coronal T1 postcontrast imaging demonstrates the presence of a peripherally enhancing, centrally necrotic space-occupying mass lesion in the left frontal lobe, with mass effect upon the left lateral ventricle and mild subfalcine herniation; (B) case 4: rounded, peripherally enhancing, centrally necrotic mass lesion in the right frontal lobe. No significant vasogenic edema is noted; (C) case 7: contrast-enhanced CT demonstrates an erosive right frontal bone metastasis that erodes through the inner and outer cortical tables. There is mass effect upon the right frontal lobe; (D) case 12: Axial T2 image demonstrates the presence of a rounded left cerebellar lesion with surrounding vasogenic edema. There is mass effect upon the lateral margins of the 4th ventricle; (E) case 13: Axial T2 image demonstrates lobulated thickening of the posterior-lateral margins of the left retina.

also had extracranial metastatic lesions, while $9(40.9 \%)$ had solitary brain metastases without evidence of other sites of metastases. Among 17 patients with available data on the onset of brain metastases relative to the original diagnosis of TETs, 10 (58.8\%) patients had metachronous brain metastases and $7(41.2 \%)$ cases of brain metastases were discovered synchronously. Surgical resection of metastatic brain lesions was the mainstay of treatment, which was supplemented by radiotherapy in some cases. One patient who had multiple intracranial lesions was treated with
WBRT and SRS. Although many patients had no local recurrence after treatment of brain metastasis, four patients with thymic carcinoma died shortly after treatment of brain metastases.

\section{Discussion}

There are limited data about the incidence of brain metastasis in patients with TETs. In a case series, Kong and colleagues reported that thymic carcinoma and thymic 

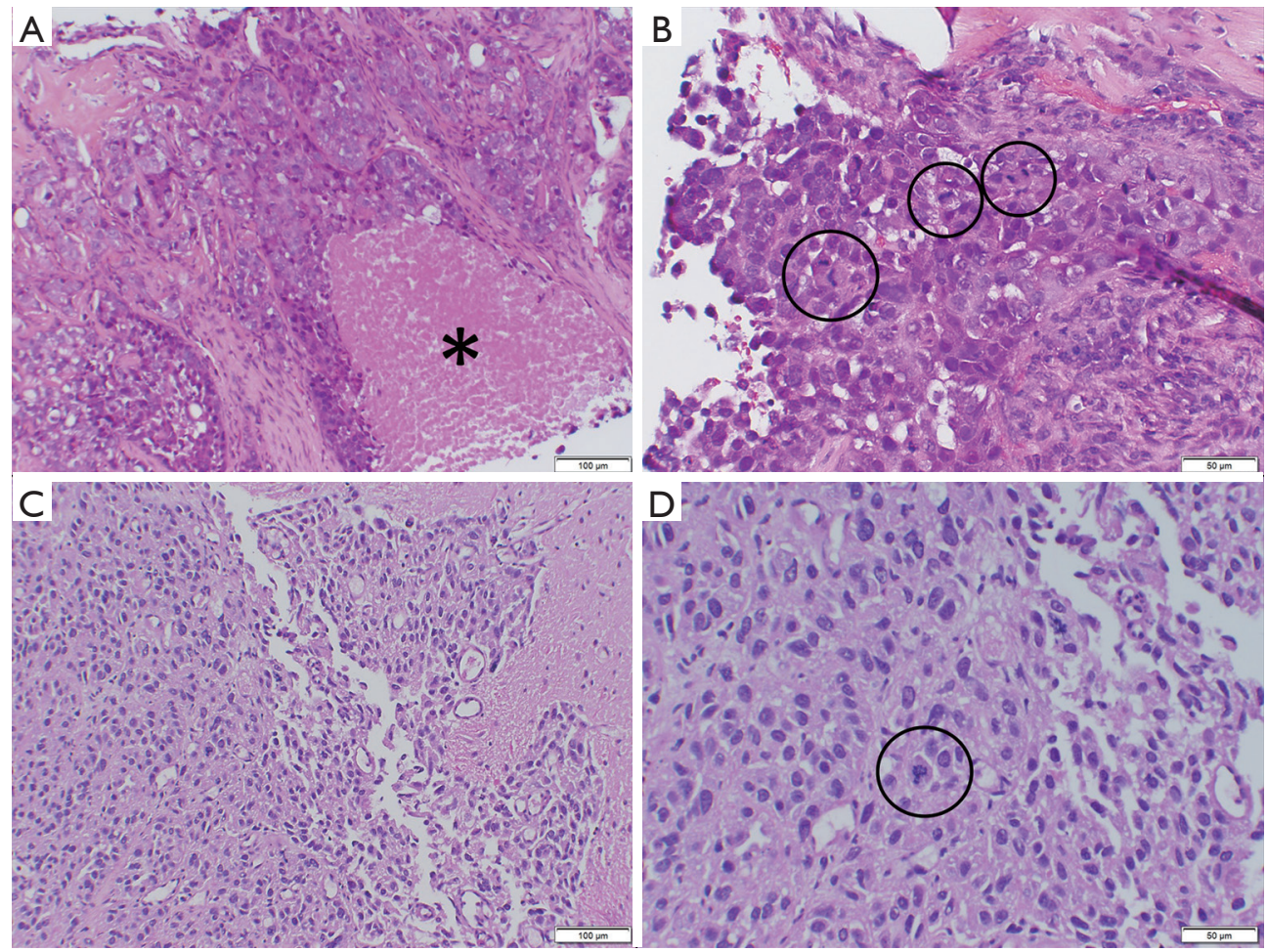

Figure 2 Histologic findings of primary tumor and brain metastasis in a case of poorly differentiated non-keratinizing carcinoma of the thymus. (A) Mediastinal mass biopsy shows nests and solid sheets of atypical epithelioid cells and necrosis (asterisk); (B) the epithelioid tumor cells show nuclear enlargement with size variation, hyperchromasia, and frequent mitotic figures (circles); (C) resected brain metastasis consists of highly cellular proliferation of atypical epithelial cells. The tumor is relatively well demarcated, but focally infiltrative into adjacent brain parenchyma (right bottom); (D) tumor cells in the brain have morphologic features similar to those of the primary mediastinal tumor. Mitotic figures are easily found (circles). H \& E stains (A-D).

carcinoid tumors metastasize frequently to the brain, with an incidence of $17.6 \%$ and $33.3 \%$, respectively (26). In a retrospective analysis of 77 patients with TETs (with a median time from surgery resection of the primary tumor to last follow-up 57 months), 24 (31\%) developed recurrence or metastasis of TETs and only one patient with thymic carcinoma developed brain metastasis (27). Our systematic review revealed only 22 cases of brain metastases reported in the literature in patients with TETs. In most of these cases, the metastases were within the brain parenchyma (intra-axial), although extradural extension of thymic metastases invading the skull and mimicking meningiomas (extra-axial) is also described (23). The appearance of thymic metastases can vary from solid to cystic, and depending on size and location, the tumors can cause various symptoms. Complications may include hemorrhage (22) or extracranial extension (21). As expected, thymic carcinoma, the most aggressive form of TETs, was the most common subtype associated with brain metastases, but cases of brain metastases from less aggressive subtypes such WHO subtypes $\mathrm{A}, \mathrm{AB}$, and $\mathrm{B} 1$ thymoma were also reported $(11,12,17)$.

Consistent with the findings reported by Kong and colleagues, most of our patients were diagnosed with brain metastases after metastases had developed at extrathoracic sites, primarily bones, lungs and liver. Most of the intracranial tumors in our patients appeared as ring-enhancing, intra-axial masses on brain imaging, like previous reports by others; only in one case there was a tumor found extra-axially compressing the skull (Figure 1C). At diagnosis of intra-cranial involvement, among patients with brain imaging available for review, six patients had a solitary brain metastasis and two patients had multiple metastases. 

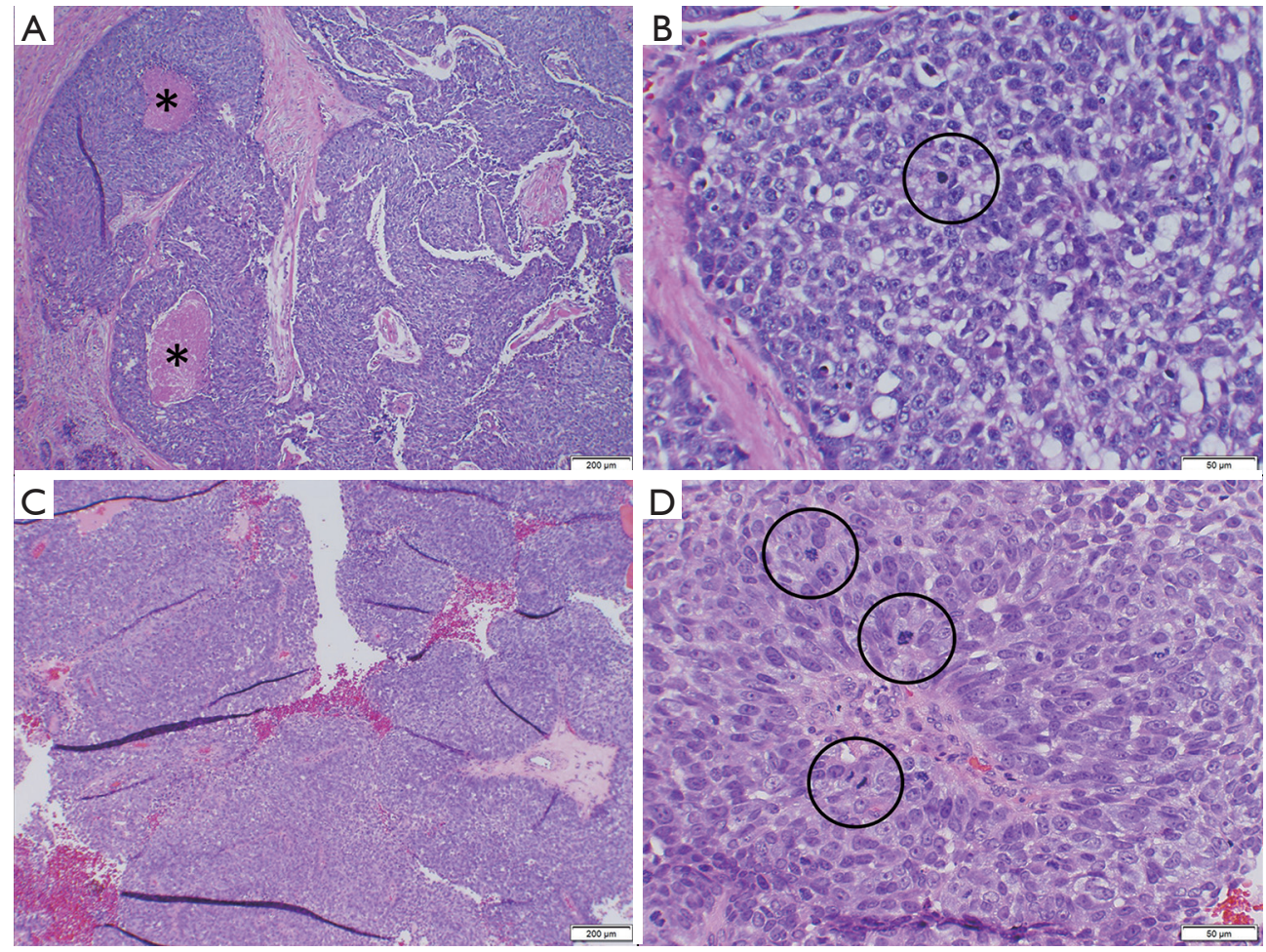

Figure 3 Pathologic features of primary tumor and brain metastasis in a case of poorly differentiated non-keratinizing carcinoma of the thymus. (A) Thymic mass excision shows anastomosing solid sheets and infiltrating small nests of highly cellular tumor cells with necrosis (asterisks); (B) tumor cells are epithelioid and have large hyperchromatic nuclei with prominent nucleoli and frequent mitotic figures (circles); (C) resected brain metastasis reveals highly cellular proliferation of tumor cells in solid sheets; (D) tumor cells in the brain have morphologic features similar to those of the primary thymic tumor. Mitotic figures are easily found (circles). Immunohistochemical features of the brain metastasis are also similar to the primary tumor with tumor cells expressing CK 5/6, CD5, and p63 (not shown). H\&E stains (A-D).

Commonly, single or oligometastatic (1-3 brain metastases) brain involvement of solid tumors is treated with surgical resection of the lesion(s) followed by WBRT, SRS followed by WBRT, or SRS alone. WBRT as the only treatment modality for limited metastatic lesions has been reported in two studies to be associated with shorter survival times, more recurrences of cancer in the brain and worse quality of life, when compared with WBRT combined with another modality to treat the brain metastasis $(28,29)$. In most of the cases of TETs metastasizing to the brain described in the literature, surgical resection alone or in combination with radiotherapy was the main modality of treatment. While a significant portion of patients achieved long term remissions, there were a few patients who died shortly after local therapy. As with other tumor types, careful selection of patients for surgical resection and/or radiotherapy is important, although the optimal treatment for brain metastases from TETs has not yet been determined.

While our report does not provide any information on the incidence rate of brain metastasis in TETs, the frequency of intracranial metastases in these patients is likely to be higher than commonly assumed. Based on our experience, we suggest that imaging studies should be performed as clinically indicated in patients with TETs, especially in patients with thymic carcinoma. Not uncommonly, patients with TETs are found to have solitary brain metastasis and these patients had a better prognosis than patients with multiple brain metastases. These findings when coupled with the observation that patients with TETs have a better prognosis when compared stage-for-stage with many solid tumors (30), appear to support a role for aggressive treatment of brain metastases in patients with TETs. 


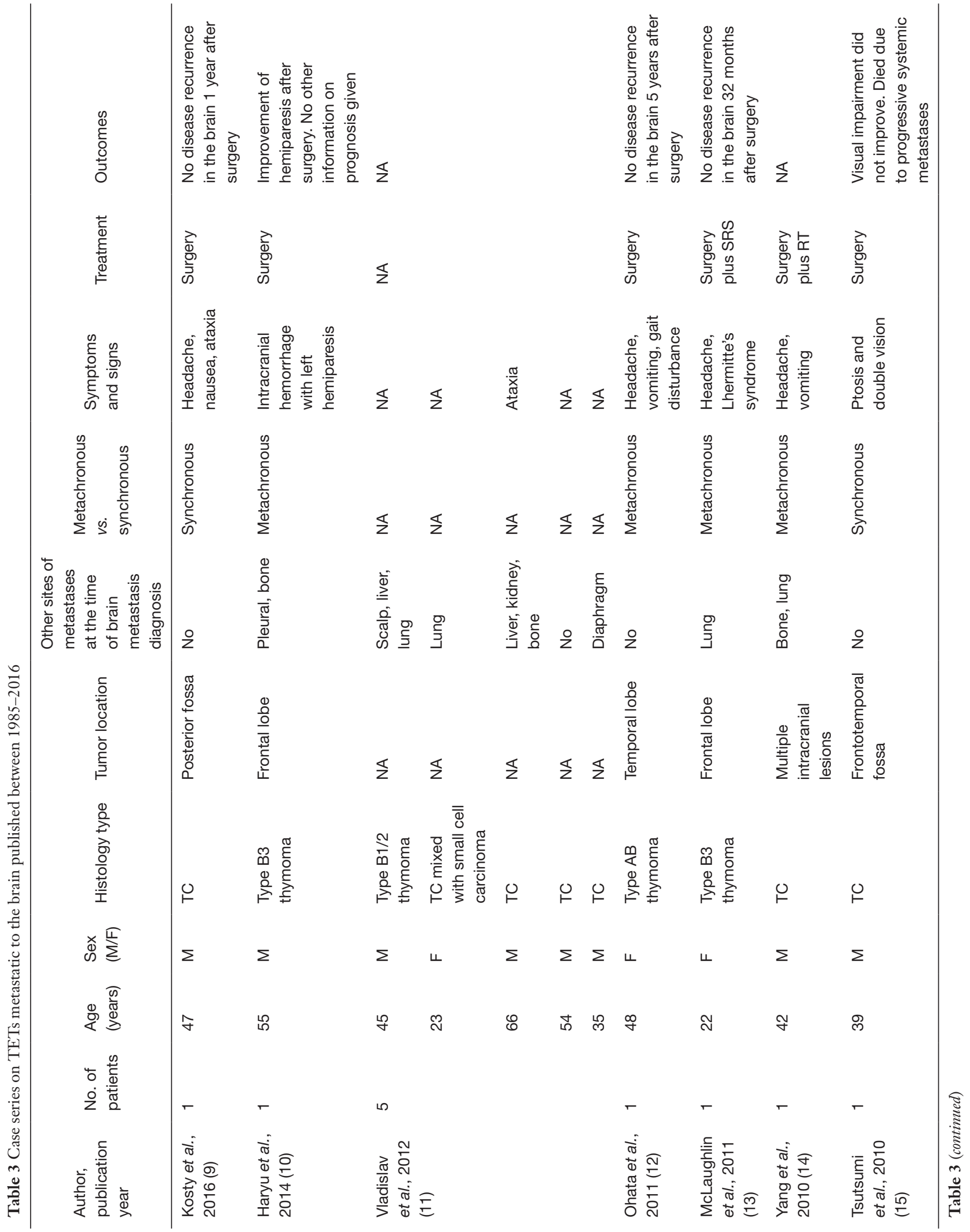




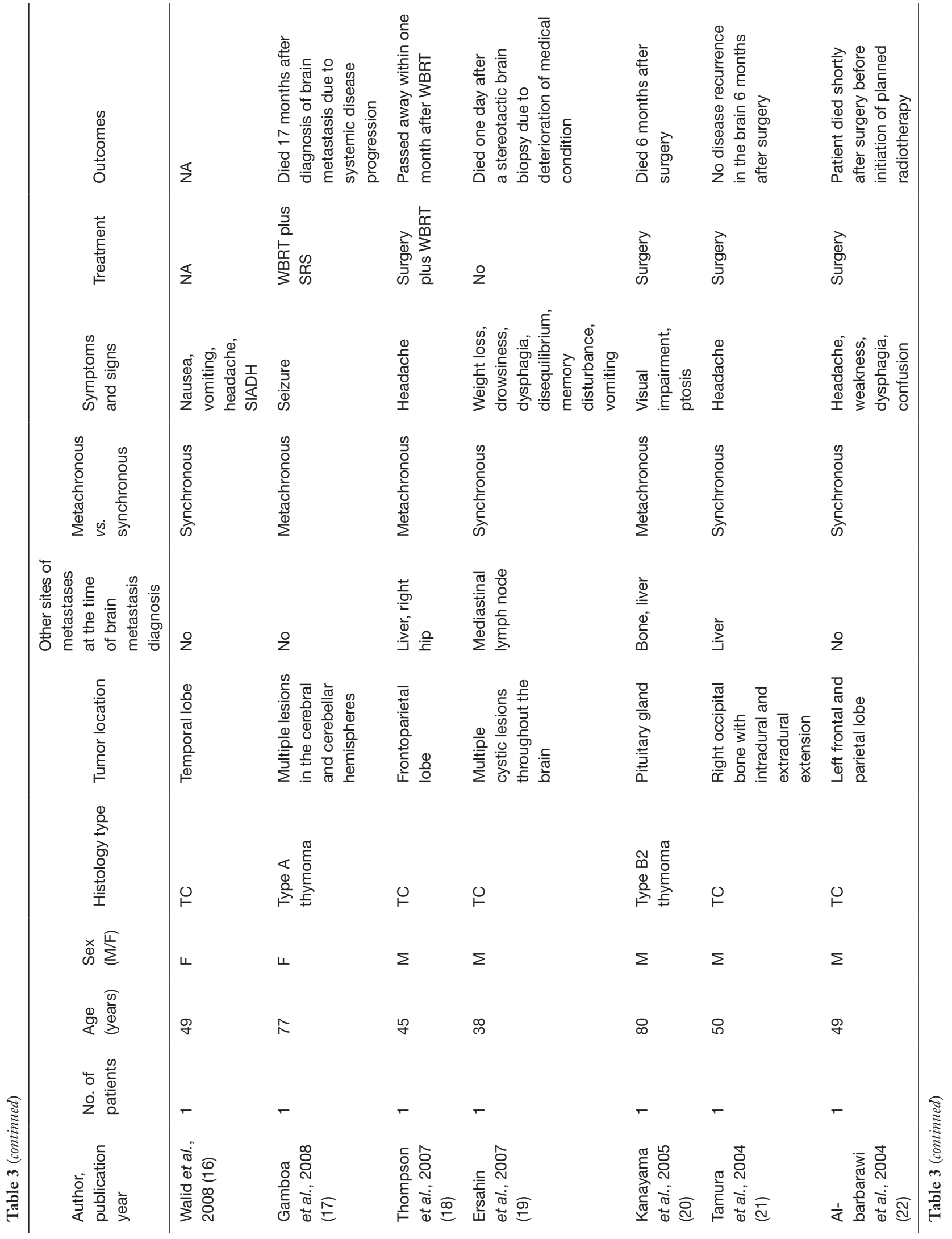




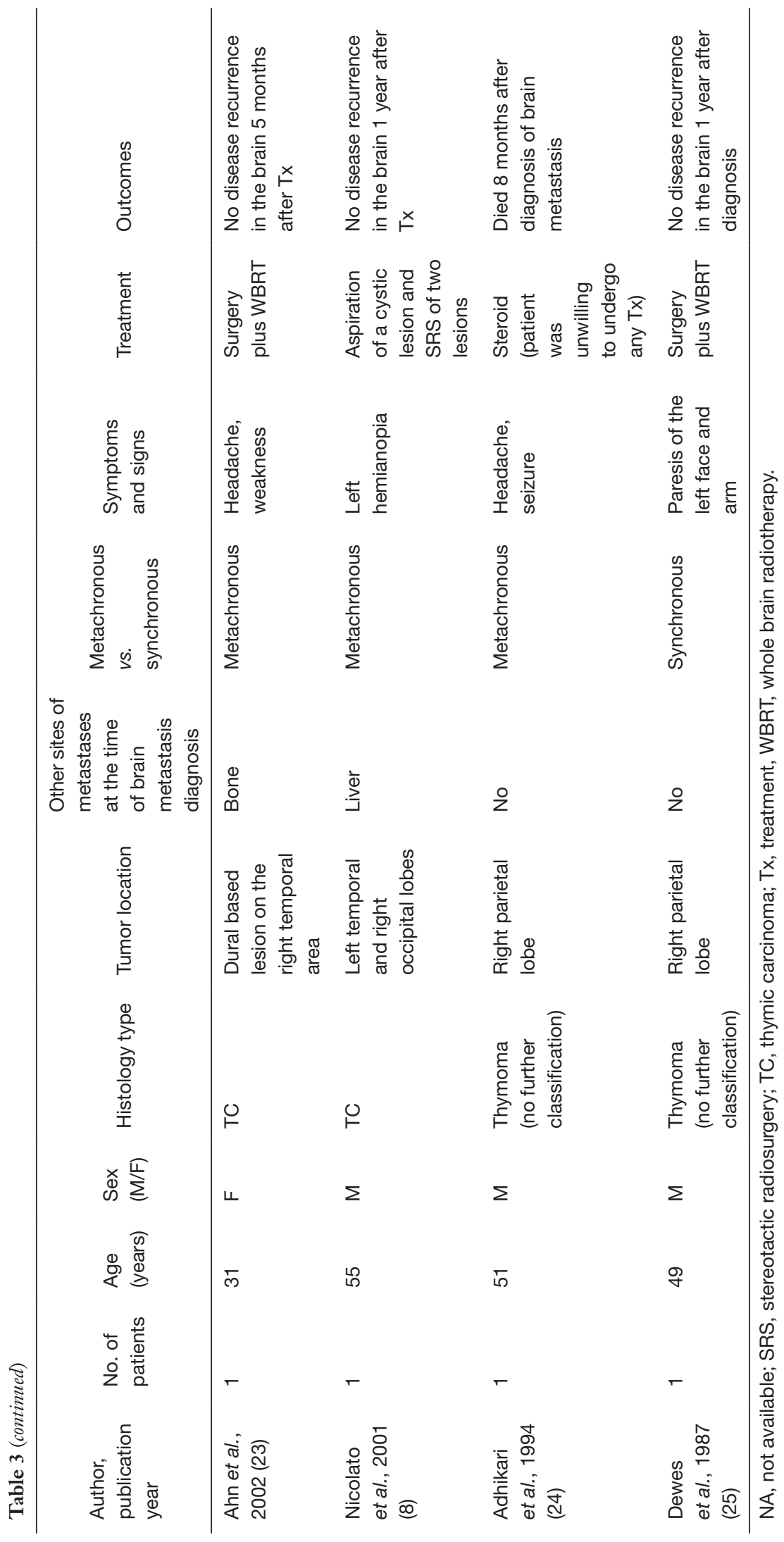




\section{Acknowledgements}

This research was supported in part by the Intramural Research Program of the NIH, National Cancer Institute, Center for Cancer Research.

\section{Footnote}

Conflicts of Interest: The authors have no conflicts of interest to declare.

Ethical Statement: All patients provided informed consent for participation in one or more clinical trials (NCT00026754, NCT01306045, NCT01621568, NCT02146170) approved by the institutional review board at the National Cancer Institute (NCI clinical trial ID: 01-C-0129, 11-C-0096, 12C-0118, 14-C-0105).

\section{References}

1. Wick MR, Scheithauer BW, Weiland LH, et al. Primary thymic carcinomas. Am J Surg Pathol 1982;6:613-30.

2. Engels EA, Pfeiffer RM. Malignant thymoma in the United States: demographic patterns in incidence and associations with subsequent malignancies. Int $\mathrm{J}$ Cancer 2003;105:546-51.

3. Nickels J, Franssila K. Thymoma metastasizing to extrathoracic sites. A case report. Acta Pathol Microbiol Scand A 1976;84:331-4.

4. Suster S, Rosai J. Thymic carcinoma. A clinicopathologic study of 60 cases. Cancer 1991;67:1025-32.

5. Fabi A, Felici A, Metro G, et al. Brain metastases from solid tumors: disease outcome according to type of treatment and therapeutic resources of the treating center. J Exp Clin Cancer Res 2011;30:10.

6. Nussbaum ES, Djalilian HR, Cho KH, et al. Brain metastases. Histology, multiplicity, surgery, and survival. Cancer 1996;78:1781-8.

7. Lagerwaard FJ, Levendag PC, Nowak PJ, et al. Identification of prognostic factors in patients with brain metastases: a review of 1292 patients. Int J Radiat Oncol Biol Phys 1999;43:795-803.

8. Nicolato A, Ferraresi P, Bontempini L, et al. Multiple brain metastases from "lymphoepithelioma-like" thymic carcinoma: a combined stereotactic-radiosurgical approach. Surg Neurol 2001;55:232-4.

9. Kosty JA, Andaluz N. Metastatic Thymic Carcinoma Presenting as a Posterior Fossa Mass: Case Report and Review of the Literature. World Neurosurg 2016;93:486.e1-6.

10. Haryu S, Saito A, Inoue M, et al. Brain metastasis from invasive thymoma mimicking intracerebral hemorrhage: case report. Neurol Med Chir (Tokyo) 2014;54:673-6.

11. Vladislav T, Jain RK, Alvarez R, et al. Extrathoracic metastases of thymic origin: a review of 35 cases. Mod Pathol 2012;25:370-7.

12. Ohata N, Usami N, Kawaguchi K, et al. Type AB thymoma with brain metastasis: Report of a case. Surg Today 2011;41:1436-8.

13. McLaughlin SS, Peckham SJ, Enis JA, et al. Young woman with thymoma metastatic to the brain controlled with gross total resection and stereotactic radiosurgery, with a subsequent uncomplicated pregnancy. J Clin Oncol 2011;29:e30-3.

14. Yang JT, Chang CM, Lee MH, et al. Thymic squamous cell carcinoma with multiple brain metastases. Acta Neurol Taiwan 2010;19:41-4.

15. Tsutsumi S, Abe Y, Yasumoto Y, et al. Metastatic skull base tumor from thymic carcinoma mimicking Tolosa-Hunt syndrome. Neurol Med Chir (Tokyo) 2010;50:499-502 .

16. Walid MS, Troup EC, Robinson JS Jr. Brain metastasis from thymic carcinoma in association with SIADH and pituitary enlargement: a case report. South Med J 2008;101:764-6.

17. Gamboa EO, Sawhney V, Lanoy RS, et al. Widespread metastases after resection of noninvasive thymoma. J Clin Oncol 2008;26:1752-5.

18. Thompson EM, Sather MD, Reyes CA, et al. Intracranial leptomeningeal metastasis from thymic carcinoma: case report and review. Surg Neurol 2007;68:233-8.

19. Ersahin M, Kilic K, Gogusgeren MA, et al. Multiple brain metastases from malignant thymoma. J Clin Neurosci 2007;14:1116-20.

20. Kanayama S, Matsuno A, Nagashima T, et al. Symptomatic pituitary metastasis of malignant thymoma. J Clin Neurosci 2005;12:953-6.

21. Tamura Y, Kuroiwa T, Doi A, et al. Thymic carcinoma presenting as cranial metastasis with intradural and extracranial extension: case report. Neurosurgery 2004;54:209-11; discussion 211-2.

22. Al-Barbarawi M, Smith SF, Sekhon LH. Haemorrhagic brain metastasis from a thymic carcinoma. J Clin Neurosci 2004;11:190-4.

23. Ahn JY, Kim NK, Oh D, et al. Thymic carcinoma with brain metastasis mimicking meningioma. J Neurooncol 2002;58:193-9.

24. Adhikari MR, Pereira P, Ramesh M, et al. Malignant 
thymoma with cerebral metastases in association with pure red cell aplasia. J Assoc Physicians India 1994;42:245-6.

25. Dewes W, Chandler WF, Gormanns R, et al. Brain metastasis of an invasive thymoma. Neurosurgery 1987;20:484-6.

26. Kong DS, Lee JI, Nam DH, et al. Cerebral involvement of metastatic thymic carcinoma. J Neurooncol 2005;75:143-7.

27. Khandelwal A, Sholl LM, Araki T, et al. Patterns of metastasis and recurrence in thymic epithelial tumours: longitudinal imaging review in correlation with histological subtypes. Clin Radiol 2016;71:1010-7.

Cite this article as: Gharwan $\mathrm{H}, \mathrm{Kim} \mathrm{C}$, Thomas A, Berman A, Kim SA, Biassou N, Steinberg SM, Rajan A. Thymic epithelial tumors and metastasis to the brain: a case series and systematic review. Transl Lung Cancer Res 2017;6(5):588-599. doi: 10.21037/ tlcr.2017.08.06
28. Patchell RA, Tibbs PA, Walsh JW, et al. A randomized trial of surgery in the treatment of single metastases to the brain. N Engl J Med 1990;322:494-500.

29. Andrews DW, Scott CB, Sperduto PW, et al. Whole brain radiation therapy with or without stereotactic radiosurgery boost for patients with one to three brain metastases: phase III results of the RTOG 9508 randomised trial. Lancet 2004;363:1665-72.

30. Okumura M, Ohta M, Tateyama H, et al. The World Health Organization histologic classification system reflects the oncologic behavior of thymoma: a clinical study of 273 patients. Cancer 2002;94:624-32. 